PHYSICAL REVIEW A 92, 029902(E) (2015)

\title{
Erratum: Analytic results for the Casimir free energy between ferromagnetic metals [Phys. Rev. A 91, 032119 (2015)]
}

\author{
G. L. Klimchitskaya and C. C. Korikov
}

(Received 23 July 2015; published 4 August 2015)

DOI: 10.1103/PhysRevA.92.029902

PACS number(s): $12.20 . \mathrm{Ds}, 42.50 . \mathrm{Lc}, 42.50 . \mathrm{Nn}, 99.10 . \mathrm{Cd}$

There are several typographical errors in this paper which have no impact on the obtained results.

Thus, the quantity $\Lambda$ on the right-hand side of Eq. (47) should be replaced with $3 \Lambda^{2}$. As a result, Eq. (48) takes the form

$$
a<\frac{3 \lambda_{p} \mu_{0}^{3 / 2} \zeta_{R}(3)}{\pi^{3}}
$$

Then, for two Ni plates, the right-hand side of this equation should be equal to $34 \mu \mathrm{m}$, not to $0.9 \mathrm{~mm}$, as is indicated in the text. The respective entropy at $T=0$ is positive from approximately 2 to $34 \mu \mathrm{m}$. 